\title{
EXPERIÊNCIA DE IMPLANTAÇÃO DO PROCESSO DE ENFERMAGEM UTILIZANDO OS DIAGNÓSTICOS DE ENFERMAGEM (TAXIONOMIA DA NANDA), RESULTADOS ESPERADOS, INTERVENÇÕES E PROBLEMAS COLABORATIVOS*
}

Maria Helena Baena de Moraes Lopes**

\section{INTRODUÇÃO}

HORTA $^{6}$, baseando-se na teoria da motivação humana de Maslow propôs o processo de enfermagem em seis fases, sendo o diagnóstico uma destas fases. Entretanto, observou que, na aplicação do processo de enfermagem, o enfermeiro encontra uma grande dificuldade para estabelecer o diagnóstico e atribuiu como causas o desconhecimento dos sintomas, das necessidades básicas alteradas e da nomenclatura destas necessidades, entre outras ${ }^{4}$. MARIA \& ARCURI ${ }^{11}$ apontaram outros fatores tais como: prática da enfermagem geralmente vinculada ao cumprimento de atividades burocráticas e técnicas, em detrimento do processo de enfermagem; reprodução do modelo biomédico no ensino de enfermagem que é baseado em patologias e execução de técnicas, no qual o aluno vê o processo de enfermagem como um mero exercício acadêmico e resistência das enfermeiras em adotar esse método de trabalho o que acaba por influenciar toda a equipe de enfermagem.

O uso dos diagnósticos de enfermagem têm provocado calorosos debates. Alguns enfermeiros julgam desnecessária sua utilização no planejamento das ações de enfermagem, desconsiderando que as intervenções devem ser justificadas pela presença de fenômenos identificáveis e avaliadas com base nos resultados esperados. Concordamos com CARPENITO ${ }^{1}$ que "os diagnósticos de enfermagem proporcionam à enfermagem uma estrutura para a organização de sua ciência.", mas que o seu uso requer conhecimento e envolve responsabilidade.

O uso dos diagnósticos de enfermagem pode trazer benefícios não só para o profissional e cliente, como também para a instituição. Segundo MILLER ${ }^{12}$, para que a enfermeira assista adequadamente o cliente, é necessário conhecer os problemas que ele está experienciando e a falta de clareza na sua identificação implica em perda de tempo e de energia, ou mesmo, de dinheiro. Para ela o objetivo da enfermagem é prover uma assistência que atenda às necessidades do cliente, ao passo que o da instituição é prestar um serviço efetivo e eficiente. Portanto, o uso dos diagnósticos de enfermagem beneficia a ambos, porque direciona a assistência de enfermagem para as necessidades de cada cliente, facilita a escolha de intervenções mais adequadas, registra de forma objetiva as reações do cliente e permite subseqüente avaliação dos cuidados de enfermagem.

O Centro de Assistência Integral à Saúde da Mulher (CAISM), da cidade de Campinas, SP, Brasil, é um hospital-escola de cerca de 150 leitos voltado para o atendimento em quatro diferentes especialidades: Ginecologia, Obstetrícia, Oncologia Ginecológica e Neonatologia. Em 1990, iniciou-se a utilização dos diagnósticos de enfermagem, segundo a taxionomia da North American Nursing Diagnosis Association (NANDA), no ensino de graduação em enfermagem ${ }^{9} \mathrm{e}$, posteriormente, no Serviço de Neonatologia do CAISM. Atualmente, encontra-se em fase de implantação em todos os setores a SAE com as seguintes fases: coleta de dados, diagnóstico (diagnóstico de enfermagem e complicações potenciais, segundo a proposta de CARPENITO $^{1}$ ), planejamento (estabelecimento das intervenções de enfermagem e dos resultados esperados), implementação e evolução. Foram utilizadas

* Trabalho apresentado no $2^{\text {nd }}$ European Conference of the Association for Common European Nursing Diagnoses, Interventions and outcomes (ACENDIO), realizado em Veneza, Itália

** Professor Assistente Doutor, Coordenadora de Graduação do Departamento de Enfermagem da Faculdade de Ciências Médicas (FCM) da Universidade Estadual de Campinas (UNICAMP) e Enfermeira do Programa de Educação Continuada do Centro de Atenção Integral à Saúde da Mulher (CAISM) - UNICAMP 
diversas estratégias para a capacitação das enfermeiras e elaborados novos modelos de impressos mais adequados para a coleta e registro dos dados. Neste trabalho relatamos as estratégias utilizadas, dificuldades e resultados obtidos.

\section{ESTRATÉGIAS UTILIZADAS NA IMPLANTAÇÃO DO PROCESSO DE ENFERMAGEM}

Desde 1991 o Programa de Educação Continuada do CAISM procurou capacitar as enfermeiras para a implantação do processo de enfermagem através de: oficinas de trabalho sobre consulta e diagnóstico de enfermagem; capacitação em consulta de enfermagem à mulher, por meio de estágio prático supervisionado em unidade básica de saúde e cursos sobre planejamento familiar; grupos de estudo sobre os diagnósticos de enfermagem e discussão de diagnósticos freqüentes na assistência à saúde da mulher e recém-nascido tais como: dor, risco para infecção, amamentação ineficaz, processo familiar alterado e disfunção sexual; discussão sobre comunicação formal, uso inadequado de siglas e abreviaturas e normatização das anotações de enfermagem; realização de pesquisas retrospectivas utilizando dados do prontuário das clientes para identificação dos diagnósticos mais freqüentes na área de Oncologia, Ginecologia, Obstetrícia (gravidez de alto risco) e Neonatologia $2,7,8,10,14,15$.

Em fevereiro de 1997 a utilização do processo de enfermagem foi considerado como meta prioritária e se iniciou um movimento para a sua implantação em todas as unidades ${ }^{3}$ através da formação de uma Comissão composta por enfermeiras do Programa de Educação Continuada, supervisoras e(ou) diretoras de serviço.

Realizou-se visitas a hospitais com experiência na utilização do processo e diagnóstico de enfermagem (Hospital Dante Pazzanese e Hospital São Paulo) e decidiu-se, então, que sua implantação ocorreria inicialmente em uma unidade piloto, a Unidade de Internação do Serviço de Oncologia.

Para capacitar as enfermeiras foram desenvolvidos três cursos sobre Processo de Enfermagem, quatro cursos sobre semiologia (dois de Semiologia Geral e dois de Semiologia do RecémNascido) ${ }^{3}$ e um curso sobre interpretação de exames laboratoriais. Todos os serviços receberam artigos sobre avaliação e controle da dor aguda e foi ministrado um curso sobre este assunto.

O livro "Diagnósticos de enfermagem: aplicação à prática clínica”, de Lynda Juall Carpenito ${ }^{1}$, traduzido para o português, foi adotado por todos os serviços como livro-texto para leitura e consulta. Periodicamente são realizadas reuniões com as enfermeiras dos diferentes serviços para discussão de textos, apresentação de estudos de casos e elaboração de impressos de coleta de dados, registro dos diagnósticos, resultados esperados, prescrição de enfermagem, evolução e anotações de enfermagem. No período noturno, os enfermeiros supervisores inicialmente realizaram reuniões com enfermeiras de todos os serviços para estudo, leitura de textos e discussão da assistência. A enfermeira do Programa de Educação Continuada, coordenadora da Comissão, reune-se mensalmente com as enfermeiras do noturno para a elucidação de dúvidas e discussão de propostas de trabalho.

Após cerca de um ano do início do processo de implantação, verificou-se a necessidade de atuar em situações clínicas não abrangidas pelos diagnósticos de enfermagem. Passamos, então, a trabalhar com os problemas colaborativos, de acordo com a proposta de CARPENITO $^{1}$. Essa autora, em 1983, propôs o modelo bifocal de prática clínica que identifica as duas situações clínicas nas quais a enfermeira intervém - uma em que principalmente prescreve ações (diagnósticos de enfermagem) e a outra em que colabora com as outras disciplinas (problemas colaborativos). "Problemas colaborativos são certas complicações fisiológicas que as enfermeiras monitoram para detectar o estabelecimento ou a modificação subseqüente em seu estado. As enfermeiras controlam os problemas colaborativos usando intervenções prescritas pelo médico e (ou) ela própria, para minimizar as complicações dos eventos"

\section{RESULTADOS OBTIDOS}

De modo geral, as enfermeiras têm participado regularmente das reuniões, demonstrando interesse em desenvolver suas habilidades e conhecimentos. A resistência inicial das enfermeiras está sendo gradualmente superada, durante as reuniões relatam resultados positivos e demostram interesse em aperfeiçoar a sua prática. Entretanto, o envolvimento das enfermeiras não é uniforme e ainda há questionamentos quanto a demanda de tempo e validade do uso dos diagnósticos de enfermagem.

Apresentaremos, a seguir, a fase de implantação do Processo nas diferentes unidades do CAISM e as dificuldades encontradas.

1. O Serviço de Enfermagem em Neonatologia foi o primeiro a implantar o Processo de Enfermagem em 1992, com as seguintes fases: coleta de dados, 
diagnóstico, intervenções e evolução de enfermagem. Identificou-se a necessidade de envolver todo o grupo de enfermeiras, porque apenas no período da manhã são realizadas a avaliação e a prescrição, cabendo às enfermeiras dos outros turnos e à equipe de enfermagem a implementação das prescrições que, por vezes, é realizada de forma rotineira, sem envolvimento com o processo.

2. Serviço de Enfermagem em Oncologia - A Unidade de Internação iniciou a implantação do Processo em 1997 e, momento atual, são desenvolvidas todas as suas fases. Gradualmente, foi implantada o Processo em número crescente de clientes até chegar ao número total (a unidade possui 35 leitos). Encontra-se em fase de teste um formulário contendo já impressos os diagnósticos, resultados esperados e intervenções mais freqüentes.

3. Serviço de Enfermagem em Oncologia - No Ambulatório de Radioterapia foi definido o formulário de coleta de dados, contendo os diagnósticos e complicações potenciais mais freqüentes de acordo com a literatura e experiência clínica, as intervenções e os resultados esperados apresentados como "check-list".

4. Serviço de Enfermagem em Ginecologia - No Ambulatório foi elaborado um projeto para a atuação de enfermagem em grupos educativos e atendimento individual a mulheres portadoras de endometriose, utilizando o processo de enfermagem e os diagnósticos da NANDA ${ }^{13}$ e um roteiro para atendimento de mulheres vítimas de violência sexual, que inclui os diagnósticos de enfermagem mais freqüentes, de acordo com a literatura. Na Unidade de Internação, iniciou-se com a aplicação do Processo a um grupo de nove clientes, porém houve pouco envolvimento da equipe com o cumprimento da prescrição de enfermagem, o que acabou por desmotivar o grupo de enfermeiras deste Setor. Atualmente apenas a coleta de dados tem sido feita de forma sistemática e pretende-se estruturar a prescrição de enfermagem.

5. Serviço de Enfermagem em Obstetrícia - Retaguarda: a implantação ocorreu após um curso de 16 horas sobre Processo de Enfermagem e acompanhamento diário, pela enfermeira do Programa de Educação Continuada, durante as duas primeiras semanas. Todas as fases foram implantadas, mas os enfermeiros ainda têm dificuldade de realizar a evolução de enfermagem considerando os diagnósticos identificados inicialmente e os resultados esperados.

6. Serviço de Enfermagem em Obstetrícia - Unidade de Internação: esta unidade é composta por dois setores, a saber, Alojamento Conjunto e Patologia Obstétrica. Iniciou-se com a implantação do Processo junto a gestantes com patologias clínicas e obstétricas e, no momento está sendo desenvolvido no Alojamento Conjunto, onde se está testando novos impressos contendo os diagnósticos, intervenções e resultados esperados mais comuns, considerando os objetivos da assistência de enfermagem neste setor, isto é, estabelecimento do aleitamento materno, autocuidado no puerpério, orientação e cuidado com o recém-nascido. 7. Serviço de Enfermagem em Obstetrícia - Pronto Atendimento: foi elaborado um roteiro para a coleta de dados junto às parturientes admitidas para internação que inclui informações sobre experiência anterior com aleitamento materno, apoio social, nível de dor, planejamento e aceitação da gravidez.

8. Serviço de Enfermagem em Centro Cirúrgico: elaborou-se um formulário de coleta de dados, contendo impressos os possíveis diagnósticos de enfermagem e intervenções, a ser utilizado no período pré, trans e pósoperatório. Pretende-se rever o formulário elaborado e iniciar o seu uso. A maior dificuldade é o pouco tempo que a enfermeira dispõe para interagir com a cliente no período pré-operatório no Centro Cirúrgico e a inexistência de um local apropriado para a entrevista. Encontra-se em fase de discussão a coleta de dados préoperatórios pelas enfermeiras das Unidades de Internação.

\section{DISCUSSÃO}

A implantação do Processo de Enfermagem tem sido lenta e difícil. Há resistência dos enfermeiros e isto se deve muitas vezes à falta de experiência prévia em outros serviços, à visão de que o Processo é complexo, demanda muito tempo e não é, portanto, factível na prática diária.

O apoio e incentivo da diretoria de enfermagem, tanto quanto a adesão do grupo de enfermeiras, é imprescindível para que o processo se inicie e se mantenha. Em nossa experiência, inicialmente, várias estratégias de capacitação e conscientização do grupo de enfermeiras fracassaram pelo pouco envolvimento de diretoras e supervisoras com essa metodologia de assistência.

CARPENITO $^{1}$ afirma que historicamente as enfermeiras durante a sua formação aprendem a usar os diagnósticos médicos para descrever o enfoque da enfermagem e, como a terminologia médica é mais conhecida, portanto, fácil e conveniente, algumas enfermeiras têm resistido à utilização de termos mais orientados para a enfermagem.

$\mathrm{CRUZ}^{5}$ vai mais além quando discute as contribuições dos diagnósticos de enfermagem para a autonomia da enfermeira, ela afirma que "nós usamos muitas artimanhas para dissimular a necessidade de 
buscarmos pontuar mais objetivamente quais são os fenômenos clínicos que demandam cuidados de enfermagem. Enquanto não houver um acordo sobre quais sejam os focos da ciência clínica da enfermagem haverá grande dificuldade para consistentemente articularmos o desenvolvimento de um corpo de conhecimentos que sustente a disciplina enfermagem e legitime a autonomia da profissão"5.

Quando nos responsabilizamos pela assistência prestada e resultados obtidos, estimulamo-nos a adquirir novos conhecimentos e habilidades para realizarmos intervenções eficazes. Freqüentemente deparamos com desafios que exigem de nós busca de aperfeiçoamento profissional, constatamos falta de preparo em situações

\section{REFERÊNCIAS BIBLIOGRÁFICAS}

01. CARPENITO, L. J. Nursing diagnosis: application to clinical practice. 7. ed. Philadelphia: Lippincott, 1997. 1200p.

02. CARVALHO, L.H.; NOMELLINI, P.F.; FÂNCIO, S.A.; LOPES, M.H.B.de M. Diagnósticos de Enfermagem em uma Unidade de Internação de Gestante de Alto Risco segundo a Taxonomia da NANDA. In: SIMPÓSIO NACIONAL SOBRE OS DIAGNÓSTICOS DE ENFERMAGEM, 3., Fortaleza, 1996, Anais. Fortaleza: Associação Brasileira de Enfermagem - Seção - CE, 1996. p. 66/Resumo/ 03. CHRISTÓFORO, F.M.; VALE, I.N.do; LOPES, M.H.B.de M. Sistematização da assistência de enfermagem \& qualidade de assistência. J.Novos Passos, v. 10, n. 94, p. 12, out. 1997.

04. CRUZ, I.C.F.da. Diagnóstico de enfermagem e sua aplicação: revisão da literatura.Rev. Esc.Enfermagem USP, v. 24, n. 1, p. 149-162, abr. 1990.

05. CRUZ, I.C.F.da. Contribuições do diagnóstico de enfermagem para a autonomia da enfermeira. / Conferência apresentada no $3^{\circ}$ Simpósio Nacional sobre os Diagnósticos de Enfermagem, Fortaleza, 1996. 11 p./.

06. HORTA, W.de A. Histórico. In: Processo de enfermagem. São Paulo: EPU, 1979. p. 3738.

07. LOPES, M.H.B.de M.; MONTAGNOLI, E.T.de; MAIA, C.A. Diagnósticos de Enfermagem mais freqüentes em uma unidade de internação de ginecologia. Rev.latino-am.Enfermagem. v. 7 , n. 5, dez. 1999.

08. LOPES, M.H.B.de M.; TEIXEIRA, J.M.; FREITAS, M.R.R. Apoio à decisão em enfermagem nos casos de eliminação urinária alterada: Sistema Alturin Exp. Rev. Bras. Enfermagem, Brasília, v. 50, n. 2, p. 163-168, abr./jun., 1997. como ansiedade, dor e morte, passamos a questionar rotinas sedimentadas e bases não científicas de nossas ações.

A experiência vivida e aqui relatada mostrou que há necessidade de contínuo acompanhamento e utilização de diferentes estratégias para que seja efetiva e eficiente a implantação do Processo de Enfermagem. Atualmente encontra-se em discussão a implantação em nosso hospital da prescrição médica e de enfermagem informatizadas, pretendemos com base nas experiências vivenciadas, nos estudos já realizados e outros que pretendemos realizar, elaborar um modelo que inclua tanto os diagnósticos, como os resultados esperados, intervenções e problemas colaborativos. Este é um novo desafio que esperamos enfrentar com êxito.

09. LOPES, M.H.B. de M.; VALE, I.N. do; BARBOSA, M. Opinião dos alunos quanto ao ensino dos diagnósticos de enfermagem segundo a taxionomia da NANDA. Rev. Esc. Enfermagem USP, v. 31, n. 1, p. 80-88, abr. 1997.

10. LOPES, R.A.M.; MACEDO, D.D.; LOPES, M.H.B. de M. Diagnósticos de enfermagem mais freqüentes em uma unidade de internação de oncologia. Rev.latino-am.Enfermagem. v. 5, n. 4, p. 35-41, out. 1997.

11. MARIA, V.L.R.; ARCURI, E.A.de M. O ensino e a prática do diagnóstico de enfermagem em uma instituição governamental. In: SIMPÓSIO NACIONAL SOBRE DIAGNÓSTICOS DE ENFERMAGEM, 1, São Paulo, 1991. Anais. São Paulo: GIDE - SP, 1991. p. 6-45.

12. MILLER, E. How to make nursing diagnosis work: administrative and clinical strategies. Norwalk/San Mateo: Appleton \& Lange, 1989. $366 \mathrm{p}$.

13. NOMELLINI, P.F.; HIGA, R.; LOPES, M.H.B.de M. Assistência a mulheres portadoras de endometriose: proposta de atuação da enfermagem em grupos educativos e atendimento individual. In: CONGRESSO BRASILEIRO DE ENFERMAGEM, 50., Salvador, 1998. Resumos. Salvador: Associação Brasileira de Enfermagem - Seção - BA, 1998. p. 305 /Resumo/.

14. VALE, I.N.do; SOUZA, S.; NETTO, S.N.Y.; CARDOSO, S.M.S.; LOPES, M.H.B. de M. Avaliação preliminar dos diagnósticos de enfermagem numa UTI neonatal. Acta. Paul. Enfermagem, v. 9, p. 108, 1996a. Número especial./Resumo/.

15. VALE, I.N.do; SOUZA, S.; CARDOSO, S.M.; LOPES, M.H.B.de M. Utilizando a taxonomia de NANDA em uma UTI neonatal. In: SIMPÓSIO NACIONAL SOBRE OS DIAGNÓSTICOS DE ENFERMAGEM, 3., Fortaleza, 1996b. Anais. Fortaleza: Associação Brasileira de Enfermagem - Seção - CE, 1996. p. 67-68./Síntese/. 